\title{
Classical Polylogarithms for Amplitudes and Wilson Loops
}

\author{
A. B. Goncharov, ${ }^{1}$ M. Spradlin, ${ }^{2}$ C. Vergu, ${ }^{2}$ and A. Volovich ${ }^{2}$ \\ ${ }^{1}$ Department of Mathematics, Brown University, Box 1917, Providence, Rhode Island 02912, USA \\ ${ }^{2}$ Department of Physics, Brown University, Box 1843, Providence, Rhode Island 02912, USA
}

\begin{abstract}
We present a compact analytic formula for the two-loop six-particle maximally helicity violating remainder function (equivalently, the two-loop lightlike hexagon Wilson loop) in $\mathcal{N}=4$ supersymmetric Yang-Mills theory in terms of the classical polylogarithm functions $\mathrm{Li}_{k}$ with cross-ratios of momentum twistor invariants as their arguments. In deriving our formula we rely on results from the theory of motives.
\end{abstract}

\section{INTRODUCTION}

The past few years have witnessed revolutionary advances in our understanding of the structure of scattering amplitudes, especially in $\mathcal{N}=4$ supersymmetric YangMills theory (SYM). It is easy to argue that the seeds of modern progress were sown already in the 1980s with the discovery of the Parke-Taylor formula for the simplest nontrivial amplitudes: tree-level maximally helicity violating (MHV) gluon scattering. The mere existence of such a simple formula for a quantity which otherwise would have been prohibitively difficult to calculate using traditional Feynman diagram methods signalled the tantalizing possibility that a great vista of unanticipated structure in scattering amplitudes awaited exploration.

In contrast to the situation at tree level, it is fair to say that recent progress at loop level has mostly been evolutionary rather than revolutionary, driven primarily by faster computers, improved algorithms (both analytic and numeric), and software for multiloop calculations which has been made publicly available. Yet we hope that a great new vista of unexplored structure awaits us also at loop level in SYM theory.

This paper is concerned with the planar two-loop sixparticle MHV amplitude [1, 2], which in a sense is the simplest nontrivial SYM loop amplitude. The known infrared and collinear behavior of general amplitudes, conveniently encapsulated in the ABDK/BDS ansatz [3, 4], determines the $n$-particle MHV amplitude at each loop order $L \geq 2$ up to an additive finite function of kinematic invariants called the remainder function $R_{n}^{(L)}$. Given the presumption of dual conformal invariance [ $[\underline{5}, \underline{6}]$ for SYM amplitudes (not yet proven, but supported by all available evidence [1, 3, 4, 4, 8] $), R_{n}^{(L)}$ can depend on conformal cross-ratios only. Since there are no cross-ratios for $n=4,5$, the first nontrivial remainder function is $R_{6}^{(2)}$.

The same function $R_{6}^{(2)}$ is also believed $9[12$ to arise as the expectation value of the two-loop lightlike hexagon Wilson loop in SYM theory [13, 14] (after appropriate subtraction of ultraviolet divergences, e.g. [15]). Numerical agreement between the two remainder functions was established in [1, 14]. In a heroic effort, Del Duca, Duhr, and Smirnov (DDS) explicitly evaluated the appropriate
Wilson loop diagrams to obtain an analytic expression for $R_{6}^{(2)}$ as a 17-page linear combination of generalized polylogarithm functions [16, 17] (see also [18]).

The motivation for the present work is the belief that if SYM theory is really as beautiful and rich as recent developments indicate, then there must exist a more enlightening way of expressing the remainder function $R_{6}^{(2)}$. Ideally, like the Parke-Taylor formula at tree level, the expression should provide encouragement and guidance as we seek deeper understanding of SYM at loop level.

We present our new formula for $R_{6}^{(2)}$ in the next section and then describe the algorithm by which it was obtained.

\section{THE REMAINDER FUNCTION $R_{6}^{(2)}$}

The remainder function $R_{6}^{(2)}$ is usually presented as a function of the three dual conformal cross-ratios

$$
u_{1}=\frac{s_{12} s_{45}}{s_{123} s_{345}}, \quad u_{2}=\frac{s_{23} s_{56}}{s_{234} s_{123}}, \quad u_{3}=\frac{s_{34} s_{61}}{s_{345} s_{234}},
$$

of the momentum invariants $s_{i \cdots j}=\left(k_{i}+\cdots+k_{j}\right)^{2}$, though we will see shortly that cross-ratios of momentum twistor invariants are more natural variables. In terms of

$$
x_{i}^{ \pm}=u_{i} x^{ \pm}, \quad x^{ \pm}=\frac{u_{1}+u_{2}+u_{3}-1 \pm \sqrt{\Delta}}{2 u_{1} u_{2} u_{3}},
$$

where $\Delta=\left(u_{1}+u_{2}+u_{3}-1\right)^{2}-4 u_{1} u_{2} u_{3}$, we find

$$
\begin{aligned}
& R_{6}^{(2)}\left(u_{1}, u_{2}, u_{3}\right)=\sum_{i=1}^{3}\left(L_{4}\left(x_{i}^{+}, x_{i}^{-}\right)-\frac{1}{2} \operatorname{Li}_{4}\left(1-1 / u_{i}\right)\right) \\
& -\frac{1}{8}\left(\sum_{i=1}^{3} \operatorname{Li}_{2}\left(1-1 / u_{i}\right)\right)^{2}+\frac{1}{24} J^{4}+\frac{\pi^{2}}{12} J^{2}+\frac{\pi^{4}}{72} .
\end{aligned}
$$

Here we use the functions

$$
\begin{aligned}
& L_{4}\left(x^{+}, x^{-}\right)=\frac{1}{8 ! !} \log \left(x^{+} x^{-}\right)^{4} \\
& +\sum_{m=0}^{3} \frac{(-1)^{m}}{(2 m) ! !} \log \left(x^{+} x^{-}\right)^{m}\left(\ell_{4-m}\left(x^{+}\right)+\ell_{4-m}\left(x^{-}\right)\right)
\end{aligned}
$$


and

$$
\ell_{n}(x)=\frac{1}{2}\left(\operatorname{Li}_{n}(x)-(-1)^{n} \operatorname{Li}_{n}(1 / x)\right),
$$

as well as the quantity

$$
J=\sum_{i=1}^{3}\left(\ell_{1}\left(x_{i}^{+}\right)-\ell_{1}\left(x_{i}^{-}\right)\right) .
$$

Note that in the Euclidean region where all $u_{i}>0$, the $x_{i}^{+}$never enter the lower half-plane and the $x_{i}^{-}$never enter the upper half-plane. The expression (3) is valid in the Euclidean region with the understanding that the branch cuts of $\operatorname{Li}_{n}\left(x_{i}^{+}\right)$and $\operatorname{Li}_{n}\left(1 / x_{i}^{-}\right)$are taken to lie below the real axis while the branch cuts of $\operatorname{Li}_{n}\left(x_{i}^{-}\right)$and $\operatorname{Li}_{n}\left(1 / x_{i}^{+}\right)$are taken to lie above the real axis. (The quantities $x_{i}^{+} x_{i}^{-}$appearing as arguments of the logs are always positive.) In writing (3) extreme care has necessarily been taken to ensure the proper analytic structure. For example one can easily check that $J$ naively simplifies to $\frac{1}{2} \log \left(x^{-} / x^{+}\right)$, but this relation only holds in the regions $\Delta>0$ or $u_{1}+u_{2}+u_{3}<1$. We caution the reader that any attempt to use any such naive relations, including the well-known relation between $\operatorname{Li}_{n}(1 / x)$ and $\mathrm{Li}_{n}(x)$, without careful consideration of the branch structure, voids our warranty on (3).

Besides its great simplicity, two notable features of (3) which set it apart from the DDS formula are manifest symmetry under any permutation of the $u_{i}$, and the fact that the expression is valid and readily evaluated for all positive $u_{i}$, in particular also outside the unit cube.

\section{DESCRIPTION OF THE ALGORITHM}

\section{A Convenient Choice of Variables}

The DDS formula is expressed in terms of the classical polylogarithms $\mathrm{Li}_{k}$ as well as a collection of considerably more complicated multiparameter generalizations studied by one of the authors [19] and defined recursively by

$$
G\left(a_{k}, a_{k-1}, \ldots ; z\right)=\int_{0}^{z} G\left(a_{k-1}, \ldots ; t\right) \frac{d t}{t-a_{k}}
$$

with $G(z) \equiv 1$, of which the harmonic polylogarithms familiar in the physics literature 20] are special cases.

The parameters of the various transcendental functions which appear in the DDS formula involve not just the cross-ratios (11), but also the more complicated combinations $1-u_{i},\left(1-u_{i}\right) /\left(1-u_{i}-u_{j}\right), u_{i}+u_{j}, u_{j k l}^{ \pm}=$ $\frac{1-u_{j}-u_{k}+u_{l} \pm \sqrt{\Delta}}{2\left(1-u_{j}\right) u_{l}}$, and $v_{j k l}^{ \pm}=\frac{u_{k}-u_{l} \pm \sqrt{\left(u_{k}+u_{l}\right)^{2}-4 u_{j} u_{k} u_{l}}}{2\left(1-u_{j}\right) u_{k}}$. This large collection of variables is redundant in an inefficient way, with many rather complicated algebraic identities amongst them.
Our computation is greatly facilitated by a judicious choice of variables which trivializes all of these algebraic relations. We choose to express the three $u_{i}$ by six variables $z_{i}$ valued in $\mathbb{P}^{1}$ (with an $S L(2, \mathbb{C})$ redundancy) via

$$
u_{1}=\frac{z_{23} z_{56}}{z_{25} z_{36}}, \quad u_{2}=\frac{z_{16} z_{34}}{z_{14} z_{36}}, \quad u_{3}=\frac{z_{12} z_{45}}{z_{14} z_{25}},
$$

where $z_{i j}=z_{i}-z_{j}$. One virtue of these coordinates is that $\Delta$ becomes a perfect square, so that the $u_{j k l}^{ \pm}$are rational functions of the $z_{i j}$. (The $v_{j k l}^{ \pm}$completely drop out as explained in the following subsection.)

We anticipate that for general $n$ the best variables for studying the remainder function will be the momentum twistors of [21]. Indeed the $z$ variables may be thought of as a particular simplification of momentum twistors which is valid for the special case $n=6$ via the relation $\langle a b c d\rangle \propto z_{a b} z_{a c} z_{a d} z_{b c} z_{b d} z_{c d}$. In terms of momentum twistors

$$
u_{1}=\frac{\langle 1234\rangle\langle 4561\rangle}{\langle 1245\rangle\langle 3461\rangle}, \quad x_{1}^{+}=-\frac{\langle 1456\rangle\langle 2356\rangle}{\langle 1256\rangle\langle 3456\rangle}, \quad \text { etc. }
$$

\section{The Symbol of a Transcendental Function}

We define a function $T_{k}$ of transcendentality degree $k$ as one which can be written as a linear combination (with rational coefficients) of $k$-fold iterated integrals of the form

$$
T_{k}=\int_{a}^{b} d \log R_{1} \circ \cdots \circ d \log R_{k},
$$

where $a$ and $b$ are rational numbers, $R_{i}(t)$ are rational functions with rational coefficients and the iterated integrals are defined recursively by

$$
\begin{aligned}
& \int_{a}^{b} d \log R_{1} \circ \cdots \circ d \log R_{n}= \\
& \int_{a}^{b}\left(\int_{a}^{t} d \log R_{1} \circ \cdots \circ d \log R_{n-1}\right) d \log R_{n}(t) .
\end{aligned}
$$

The integrals are taken along paths from $a$ to $b$. When the $R_{i}$ are rational functions in several variables the issue of local path independence (or homotopy invariance) is important (see [22]), and we have checked that $R_{6}^{(2)}$ has this property.

A useful quantity associated with $T_{k}$ is its symbol, an element of the $k$-fold tensor product of the multiplicative group of rational functions modulo constants (see 22, sec. 3]). The symbol of the function shown in (10) is

$$
\operatorname{symbol}\left(T_{k}\right)=R_{1} \otimes \cdots \otimes R_{k},
$$

and this definition is extended to all functions of degree $k$ by linearity. 
The group property for rational functions $R_{i}$ modulo constants implies that

$$
\begin{array}{r}
R_{1} \cdots \otimes\left(R_{a} R_{b}\right) \otimes \cdots R_{k}=R_{1} \cdots \otimes R_{a} \otimes \cdots R_{k} \\
+R_{1} \cdots \otimes R_{b} \otimes \cdots R_{k} \\
R_{1} \cdots \otimes\left(c R_{a}\right) \otimes \cdots R_{k}= \\
\quad R_{1} \cdots \otimes R_{a} \otimes \cdots R_{k}
\end{array}
$$

for any constant $c$ and rational functions $R_{i}$.

The symbol of all functions appearing in the DDS expression for the remainder function $R_{6}^{(2)}$ are readily computed using the above definitions. For example the classical polylogarithm functions

$$
\operatorname{Li}_{k}(z)=\int_{0}^{z} \operatorname{Li}_{k-1}(t) d \log t, \quad \operatorname{Li}_{1}(z)=-\log (1-z)
$$

clearly have

$$
\operatorname{symbol}\left(\operatorname{Li}_{k}(z)\right)=-(1-z) \otimes \underbrace{z \otimes \cdots \otimes z}_{k-1 \text { times }} .
$$

Finally, we note that knowledge of the symbol does not uniquely determine a transcendental function; there is an ambiguity consisting in adding lower degree functions multiplied by numerical constants of the appropriate degree.

\section{Constructing a Prototype}

Our first goal is to construct a simple function which has the same symbol $S$ as that of the DDS formula. Interestingly we find that the $v_{j k l}^{ \pm}$variables completely drop out of the symbol, even before switching to the $z$ variables. Although the full expression is too lengthy to reproduce here (see [23]), we find that $S$ possesses a crucial and surprising symmetry property which in components (the subscripts here run over the 15 different $z_{i j}$ 's) reads

$$
\left[S_{a b c d}-S_{b a c d}-S_{a b d c}+S_{b a d c}\right]-(a \leftrightarrow c, b \leftrightarrow d)=0 .
$$

It is known (see 24]) that transcendental functions of degree less than four can always be expressed in terms of logarithms and the classical polylogarithm functions $\mathrm{Li}_{k}$ only. However an arbitrary function of degree four (or higher) does not have this property, so it is rather remarkable that the remainder function $R_{2}^{(6)}$ can be so expressed.

The explanation for this miracle is Conjecture 1.19 of [24], from which it follows that any symbol obeying (17) can be obtained from a function involving only logarithms and the classical polylogarithm functions $\mathrm{Li}_{k}$ with $k \leq 4$. There is however no ab initio statement one can make about the arguments of those polylogarithms; they could in principle be arbitrary algebraic functions of the variables appearing in the symbol.
The key to constructing the function from the knowledge of its symbol lies in identifying the symmetry properties of the five kinds of terms which can appear, shown in the following table:

\begin{tabular}{c|c|c|c|c|} 
Function & $\mathrm{A} \otimes \mathrm{A}$ & $\mathrm{S} \otimes \mathrm{A}$ & $\mathrm{A} \otimes \mathrm{S}$ & $\mathrm{S} \otimes \mathrm{S}$ \\
\hline $\mathrm{Li}_{4}(x)$ & - & - & $\checkmark$ & $\checkmark$ \\
$\mathrm{Li}_{3}(x) \log (y)$ & - & - & $\checkmark$ & $\checkmark$ \\
$\mathrm{Li}_{2}(x) \mathrm{Li}_{2}(y)$ & $\checkmark$ & $\checkmark$ & $\checkmark$ & $\checkmark$ \\
$\mathrm{Li}_{2}(x) \log (y) \log (z)$ & - & $\checkmark$ & $\checkmark$ & $\checkmark$ \\
$\log (x) \log (y) \log (z) \log (w)$ & - & - & - & $\checkmark$
\end{tabular}

Here A (S) stands for antisymmetric (symmetric), and a checkmark under $\mathrm{A} \otimes \mathrm{A}$ indicates that the function's symbol contains a piece which is antisymmetric under exchange of the first two arguments and antisymmetric under exchange of the last two arguments, etc. The function $\log (x) \log (y) \log (z) \log (w)$ has a fully symmetric symbol and hence only contributes to $\mathrm{S} \otimes \mathrm{S}$.

The above table suggests the procedure for constructing a function with the desired symbol $S$. First we isolate the $\mathrm{A} \otimes \mathrm{A}$ part of $S$ and write down a linear combination of terms of the form $\mathrm{Li}_{2}(x) \mathrm{Li}_{2}(y)$ with that symbol, which is only possible if (17) is satisfied. After subtracting the symbol of this function from $S$ we isolate the $S \otimes A$ part which we fit with functions of the form $\mathrm{Li}_{2}(x) \log (y) \log (z)$. At the next step, $\mathrm{A} \otimes \mathrm{S}$, we need both $\mathrm{Li}_{4}(x)$ and $\mathrm{Li}_{3}(x) \log (y)$, which can be isolated from each other because the former does not contribute to the part of the symbol which is antisymmetric under exchange of the middle two arguments, as evident in (16). Ultimately we are left with terms which are completely symmetric under exchange of all arguments and can be fit via functions of the form $\log (x) \log (y) \log (z) \log (w)$.

This procedure leads to a function of degree 4 whose symbol matches that of the DDS function using only classical polylogarithm functions. One interesting feature which emerges is that the arguments of all polylogarithms in the result are either "diagonal" cross-ratios such as (8), which omit two diagonally opposite $z$ 's, or "edge" cross-ratios, which omit two neighboring $z$ 's, such as

$$
\begin{array}{ll}
x_{1}^{-}=-\frac{z_{14} z_{23}}{z_{12} z_{34}}, \quad x_{2}^{-}=-\frac{z_{16} z_{25}}{z_{12} z_{56}}, \quad x_{3}^{-}=-\frac{z_{36} z_{45}}{z_{34} z_{56}}, \\
x_{1}^{+}=-\frac{z_{14} z_{56}}{z_{16} z_{45}}, \quad x_{2}^{+}=-\frac{z_{25} z_{34}}{z_{23} z_{45}}, \quad x_{3}^{+}=-\frac{z_{12} z_{36}}{z_{16} z_{23}} .
\end{array}
$$

\section{Fitting the Remainder Function $R_{6}^{(2)}$}

The prototype for $R_{6}^{(2)}$ is incomplete in two related aspects. First of all there is considerable ambiguity in the placement of branch cuts, which the symbol (since it involves only rational functions) is completely insensitive 
to. For example one may be tempted to use the identity

$$
\prod_{i=1}^{3} \frac{1-x_{i}^{+}}{1-x_{i}^{-}}=\left(x^{+} / x^{-}\right)^{2}
$$

to replace $J$ by $\frac{1}{2} \log \left(x^{-} / x^{+}\right)$as mentioned above, but the two quantities have different branch structures in $u$ space. All such ambiguities are resolved by the physical requirement that the remainder function should be realvalued and smooth in the Euclidean region.

Secondly it remains to determine the terms in (3) proportional to $\pi^{2}$ or $\pi^{4}$, which do not contribute to the symbol. These terms can be fixed by several considerations such as checking the various Regge limits which were tabulated in [17] (see also [25 29]), the requirement that $R_{6}^{(2)}$ must vanish in the collinear limit $R_{6}^{(2)}(1-u, u, 0)=0$, or by numerically fitting to the DDS formula. We have also checked agreement with the leading term in the expansion around the collinear limit computed in [30].

\section{DISCUSSION}

We have presented in (3) a compact analytic expression for the two-loop six-particle MHV amplitude or Wilson loop remainder function $R_{6}^{(2)}$ in terms of the classical polylogarithm functions $\mathrm{Li}_{k}$ only. The simplicity of our result suggests that it is no longer outrageous to imagine the possibility of determining the remainder function for all $n$ and any number $L$ of loops, with an aim of matching onto the work of [30 34] at strong coupling.

For $n>6$ we imagine that the remainder function will continue to involve only the diagonal and edge momentum twistor cross-ratios, generalizing (8) and (18). It is also notable that our formula involves a particular combination (4) of polylogarithm functions with special analytic properties which is evidently very closely related to the Bloch-Wigner-Ramakrishnan-Zagier (BWRZ) functions (see for example [35]). These generalize very easily to any even degree $2 L$, suggesting a natural appearance in the $L$-loop remainder function. We anticipate that the consistency of collinear limits, and especially the systematic expansion away from them recently studied in [30], will tightly constrain the general form of $R_{n}^{(L)}$.

Finally we cannot pass up the opportunity to point out that the Bloch-Wigner functions (the degree 2 case of the BWRZ functions) are known [35] to compute the volumes of hyperbolic tetrahedra, raising the fascinating possibility of making a connection to recent work relating scattering amplitudes to volumes of polytopes in $\mathrm{AdS}_{5}$ [36, 37] (see also [38, 39]).

\section{Acknowledgments}

We have benefitted from stimulating discussions with N. Arkani-Hamed, J. Maldacena, and E. Witten. CV is grateful to Humboldt University for hospitality during the course of this work, which was supported in part by the DOE under contract DE-FG02-91ER40688 Tasks A (AV) and J OJI (MS), and by the NSF under grants PECASE PHY-0643150 and ADVANCE 0548311 (AV).

[1] Z. Bern, L. J. Dixon, D. A. Kosower, R. Roiban, M. Spradlin, C. Vergu and A. Volovich, Phys. Rev. D 78, 045007 (2008).

[2] F. Cachazo, M. Spradlin and A. Volovich, Phys. Rev. D 78, 105022 (2008).

[3] C. Anastasiou, Z. Bern, L. J. Dixon and D. A. Kosower, Phys. Rev. Lett. 91, 251602 (2003).

[4] Z. Bern, L. J. Dixon and V. A. Smirnov, Phys. Rev. D 72, 085001 (2005).

[5] J. M. Drummond, J. Henn, G. P. Korchemsky and E. Sokatchev, Nucl. Phys. B 826, 337 (2010).

[6] J. M. Drummond, J. Henn, G. P. Korchemsky and E. Sokatchev, Nucl. Phys. B 828, 317 (2010).

[7] F. Cachazo, M. Spradlin and A. Volovich, Phys. Rev. D 74, 045020 (2006).

[8] Z. Bern, M. Czakon, D. A. Kosower, R. Roiban and V. A. Smirnov, Phys. Rev. Lett. 97, 181601 (2006).

[9] L. F. Alday and J. M. Maldacena, JHEP 0706, 064 (2007).

[10] J. M. Drummond, G. P. Korchemsky and E. Sokatchev, Nucl. Phys. B 795, 385 (2008).

[11] A. Brandhuber, P. Heslop and G. Travaglini, Nucl. Phys. B 794, 231 (2008).

[12] N. Berkovits and J. Maldacena, JHEP 0809, 062 (2008).

[13] J. M. Drummond, J. Henn, G. P. Korchemsky and E. Sokatchev, Phys. Lett. B 662, 456 (2008).

[14] J. M. Drummond, J. Henn, G. P. Korchemsky and E. Sokatchev, Nucl. Phys. B 815, 142 (2009).

[15] C. Anastasiou, A. Brandhuber, P. Heslop, V. V. Khoze, B. Spence and G. Travaglini, JHEP 0905, 115 (2009).

[16] V. Del Duca, C. Duhr and V. A. Smirnov, JHEP 1003, 099 (2010).

[17] V. Del Duca, C. Duhr and V. A. Smirnov, JHEP 1005, 084 (2010).

[18] J. H. Zhang, arXiv:1004.1606.

[19] A. B. Goncharov, Math. Res. Lett. 5, 497 (1998).

[20] E. Remiddi and J. A. M. Vermaseren, Int. J. Mod. Phys. A 15, 725 (2000).

[21] A. Hodges, arXiv:0905.1473.

[22] A. B. Goncharov arXiv:0908.2238v2.

[23] See supplementary material at http://prl.aps.org/supplemental/PRL/v105/i15/e151605 for the symbol $S$ of $R_{6}^{(2)}$ in both $u$ and $z$ coordinates.

[24] A. B. Goncharov A. B. Goncharov, Proc. Symp. Pure Math. 55, 43 (1994).

[25] R. C. Brower, H. Nastase, H. J. Schnitzer and C. I. Tan, Nucl. Phys. B 814, 293 (2009).

[26] J. Bartels, L. N. Lipatov and A. Sabio Vera, Phys. Rev. D 80, 045002 (2009). 
[27] R. C. Brower, H. Nastase, H. J. Schnitzer and C. I. Tan, Nucl. Phys. B 822, 301 (2009).

[28] V. Del Duca, C. Duhr and E. W. N. Glover, JHEP 0812, 097 (2008).

[29] R. M. Schabinger, JHEP 0911, 108 (2009).

[30] L. F. Alday, D. Gaiotto, J. Maldacena, A. Sever and P. Vieira, arXiv:1006.2788.

[31] L. F. Alday and J. Maldacena, arXiv:0903.4707.

[32] L. F. Alday and J. Maldacena, JHEP 0911, 082 (2009).

[33] L. F. Alday, D. Gaiotto and J. Maldacena,
arXiv:0911.4708.

[34] L. F. Alday, J. Maldacena, A. Sever and P. Vieira, arXiv:1002.2459.

[35] D. Zagier, Math. Ann. 286, 613 (1990).

[36] L. Mason and D. Skinner, arXiv:1004.3498.

[37] A. Gorsky and A. Zhiboedov, J. Phys. A 42, 355214 (2009).

[38] A. Hodges, arXiv:1004.3323.

[39] Nima Arkani-Hamed, private communication. 